\title{
Electron Impact Mass Spectrometry of Alkanes in Supersonic Molecular Beams
}

\author{
Shai Dagan and Aviv Amirav \\ School of Chemistry, Sackler Faculty of Exact Sciences, Tel Aviv University, Tel Aviv, Israel
}

\begin{abstract}
The electron impact mass spectrometry of straight chain alkanes $\mathrm{C}_{8} \mathrm{H}_{18}-\mathrm{C}_{40} \mathrm{H}_{82}$, squalane, methylstearate, 1-chlorohexadecane, 1-bromohexadecane, and dioctylphthalate was studied by sampling them with supersonic molecular beams. A fly-through Brink-type electron impact ion source was used, utilizing a vacuum background ion filtration technique based on differences between the kinetic energy of the supersonic beam species and that of thermal molecules. The $70-\mathrm{eV}$ electron impact mass spectra of all the alkanes were characterized by a pronounced or dominant molecular weight peak together with all the fragment ions normally exhibited by the standard thermal 70-eV EI mass spectra. In contrast, the NIST library of most of these molecules did not show any molecular weight peak. By eliminating the intramolecular thermal vibrational energy we gained control over the degree of molecular ion fragmentation by the electron energy. At an electron energy of $18 \mathrm{eV}$ the molecular ion dissociation was further reduced considerably, with only a small absolute reduction in the peak height by less than a factor of 2 . The effect of vibrational cooling increased with the molecular size and number of atoms. Pronounced differences were observed between the mass spectra of the straight chain triacontane and its branched isomer squalane. Similar mass spectra of octacosane $\left(\mathrm{C}_{28} \mathrm{H}_{58}\right)$ achieved with $70-\mathrm{eV}$ EI in a supersonic molecular beam were obtained with a magnetic sector mass spectrometer by using an electron energy of $14 \mathrm{eV}$ and an ion source temperature of $150{ }^{\circ} \mathrm{C}$. However, this ion source temperature precluded the gas chromatography-mass spectrometry (GC-MS) of octacosane. The GC-MS of alkanes was studied with an ion trap gas chromatograph-mass spectrometer at an ion source temperature of $230{ }^{\circ} \mathrm{C}$. Thermal peak tailing was observed for $\mathrm{C}_{20} \mathrm{H}_{42}$ and heavier alkanes, whereas for $\mathrm{C}_{28} \mathrm{H}_{58}$ and heavier alkanes the severe peak tailing made quantitative GC-MS impractical. In contrast, no peak tailing existed even with $\mathrm{C}_{41} \mathrm{H}_{82}$ for GC-MS in supersonic molecular beams. The minimum detected amount of eicosane $\left(\mathrm{C}_{20} \mathrm{H}_{+2}\right)$ was shown to be $60 \mathrm{fg}$. This was demonstrated by using single ion monitoring with the quadrupole mass analyzer tuned to the molecular weight peak of $282 \mathrm{u}$. The coupling of electron impact mass spectrometry in supersonic molecular beams with hyperthermal surface ionization and a fast GC-MS inlet is briefly discussed. (f Am Soc Mass Spectrom 1995, 6, 120-131)
\end{abstract}

$\mathrm{E}$ lectron impact (EI) is the major ionization method used in mass spectrometry and gas chromatography-mass spectrometry (GC-MS). EI is a relatively simple ionization method, yet it provides high sensitivity and a uniform semiquantitative response to all molecules and atoms. In addition, the rich fragmentation pattern observed, combined with the very rich libraries of 70-eV EI mass spectra, result in substantial molecular identification and structural information capability. tions:

Despite its strength, EI suffers from several limita-

1. In many important cases the molecular weight peak is either unobserved or it is too small to be reliable.

Address reprint requests to Professor Aviv Amirav, School of Chemistry. Sackler Faculty of Exact Sciences, Tel Aviv University, Ramat Aviv 69978, Tel Aviv, Israel.
2. The ion source must be heated to reduce the response time, and the ion source metal surface can initiate thermal or catalytic molecular and molecular ion decomposition.

3. Relatively nonvolatile molecules undergo lengthy cycles of thermal adsorption-desorption in the ion source that result in gas chromatography (GC) peak tailing. Eventually, this peak tailing dictates the upper limit of molecular volatility in GC-MS.

We note that these three limitations are interrelated. The actual ion source temperature is set to a compromised value of $\sim 150{ }^{\circ} \mathrm{C}$ for direct sample insertion and $230-250{ }^{\circ} \mathrm{C}$ for GC-MS. Although increasing the ion source temperature can decrease its response time and ability to handle relatively nonvolatile molecules, it also substantially reduces the observed relative height of the molecular weight peak and promotes catalytic and thermal molecular decomposition. 
This compromise can be eliminated by the use of supersonic molecular beams [1-3] for molecular sampling in a slightly modified $\mathrm{EI}$ ion source. Supersonic molecular beams (SMB) [4-8] are characterized by unidirectional motion with controlled hyperthermal molecular kinetic energy $(0.1-30 \mathrm{eV})$, intramolecular vibrational-rotational supercooling, mass focusing as in an ideal high load jet separator, a very high flow rate in the range of $100-500 \mathrm{~mL} / \mathrm{min}$, and a convenient method for introducing samples at atmospheric pressure.

Supersonic molecular beams are used in mass spectrometry predominantly in conjunction with laserinduced multiphoton ionization followed by time-offlight mass analysis [9-14]. In these experiments the vibrational supercooling is used mostly to improve the wavelength selectivity in resonance-enhanced two or multiphoton ionization schemes.

In a supersonic molecular beam, the vibrationally supercooled molecules undergo a single passage through the "fly-through" EI ion source. Thus, the low molecular vibrational temperature is independent of the ion source temperature, the molecular weight peak height is substantially increased, and molecularthermal decomposition and adsorption-desorption on the ion-source walls is avoided.

Amirav and Danon [2] have demonstrated the considerable effect of vibrational supercooling encountered in SMB on the electron impact mass spectrometry (EI-MS) of bromopentane isomers. This effect was also studied in cholesterol [15] and various isomers of octane [3]. In these studies electrostatic background ion filtration $[2,3,16,17]$ was used. This method is based on the observation that in a seeded supersonic molecular beam, when hydrogen or helium is used as the carrier gas, heavy organic molecules acquire hyperthermal kinetic energy. The ions formed in the ion cage possess a kinetic energy that is the sum of their electrically given ion energy and their directional hyperthermal kinetic energy. Because the thermal molecules of the vacuum background have only small $(<0.1-\mathrm{eV})$ random kinetic energy, the use of a simple small retarding field along the ion trajectory results in effective background ion filtration. This effect was used for mass spectrometry of atoms and small molecules to improve the beam signal-to-vacuum background ratio $[16,17]$. We have found that this procedure could have a major impact on organic mass spectrometry for the following reasons: (1) Background ion filtration also eliminates the self-background of sample molecules scattered from the ion source and vacuum chamber walls. This self-background elimination uncovers the genuine mass spectra of the vibrationally cold molecules. (2) Eliminating the self-background also results in a very fast ion source $(<1 \mathrm{~ms}$ ) response time regardless of the molecular volatility because any surface-scattered molecule loses its kinetic energy and is filtered out after ionization. (3) Because only molecules in the SMB are analyzed, the electron emission fila- ment can be close to the ion cage and emission currents over $10 \mathrm{~mA}$ can be routinely used. Therefore, increased ionization emission current and reduced background can compensate for the loss of sensitivity associated with the fast passage and lack of multiple scattering of the molecule in the EI ion source.

Although the combination of EI and SMB already has been demonstrated, no detailed study of its manifestation in organic mass spectrometry is available. This paper presents our results from the systematic study of the homologous alkane series $\mathrm{C}_{8} \mathrm{H}_{18}-\mathrm{C}_{40} \mathrm{H}_{82}$ and their derivatives. These molecules have the same character and their size and vibrational heat capacity systematically increase. This group of molecules was chosen because it represents molecules that are important to petrochemical mass spectrometry, whose mass spectra usually do not exhibit a molecular weight peak at the $1 \%$ relative height scale. In addition, chemical ionization is less effective with alkanes both in terms of extensive fragmentation and also because of reduced sensitivity that results from their relatively small proton affinity.

\section{Experimental}

In Figure 1, the supersonic molecular beam-mass spectrometry (SMB-MS) apparatus is shown (upper), together with an expanded view of the inlet, which also serves as a fast gas chromatograph (lower). The carrier gas $\left(\mathrm{H}_{2}\right.$ or $\left.\mathrm{He}\right)$ is introduced via needle valve $V_{1}$ at a flow rate of about $120-150 \%$ above the flow required by the column. The excess gas flows to the atmosphere and serves as a "septum flush". The samples are introduced as in a direct insertion device from the lower portion of the vaporizing oven in a conventional small test tube. No air lock and bypass pumping is required, and the upper entrance remains open to the atmosphere. The temperature gradient in the inlet allows easy control of the sample vaporization rate with mass spectrometry feedback. The upper opening serves for fast GC injection [18] with conventional syringes such as the SGE $1.0-\mu \mathrm{L}$ syringe (SGE, Australia). Manual injection is fast and simple because no septum has to be penetrated. Only below a certain penetration depth of the syringe into the injector does the carrier gas vaporize the injected amount and sweep it into the column (above this level the sample is swept outside as in septum flush devices). The injector is temperature controlled up to $500{ }^{\circ} \mathrm{C}$, and the injector liner is made either of Vespel ${ }^{1 j i}$ or glass. All seals are made of graphite.

The injector is mounted on a 3/4-in. stainless steel tube that contains a $50-\mathrm{cm}-\mathrm{long}, 0.53-\mathrm{mm}-\mathrm{i} . \mathrm{d}$. megabore column [DB-1 or DB-5MS, $1.5-\mu$ film thickness ( \& W Scientific, Folsom, CA)]. The GC column is separately heated and its temperature is controlled. At a relatively high temperature it merely serves as a transfer line for the vaporized molecules. The output of the column is mixed with make up gas (helium or 

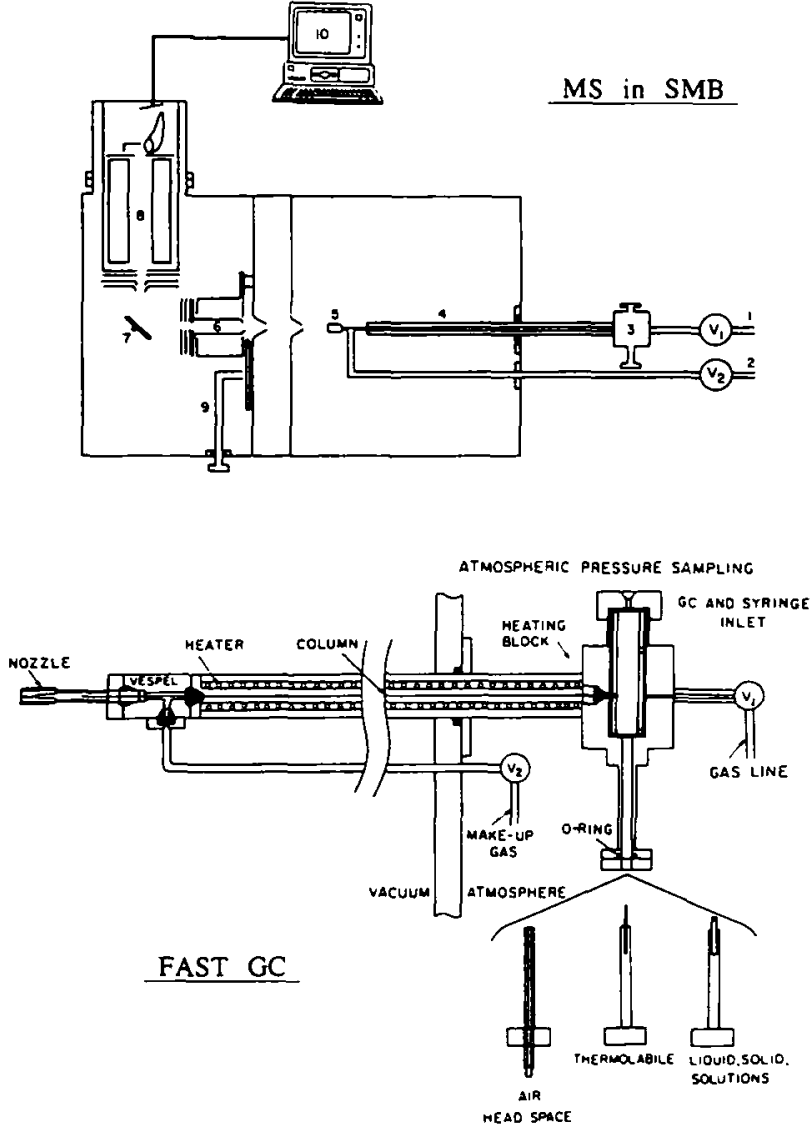

Figure 1. The sample inlet and mass spectrometer apparatus. The carrier gas flow $\mathrm{He}$ or $\mathrm{H}_{2}$, is controlled by needle valves (1) and (2). The sample is injected or introduced into the vaporizing oven (3) and flows through the temperature-controlled capillary column (4). The gas mixture expands through a supersonic nozzle (5) and is double differentially pumped. The sample molecules are ionized in the EI ion source (6), deflected by $90^{\circ}$ by the ion deflector (7) and mass analyzed by the quadrupole mass spectrometer (8). The molecular beam also can be square wavemodulated by the beam chopper (9). The data are processed and presented on a PC (10). The lower section shows an expanded view of the fast gas chromatograph and sample inlet.

hydrogen) provided by needle valve $V_{2}$. This needle valve controls the pressure behind the nozzle and thus affects the flow rate through the column. When $V_{2}$ is closed the linear velocity of the carrier gas is about $2000 \mathrm{~cm} / \mathrm{s}$, whereas when it is open the column flow rate can be regulated to any value, including a reverse protective flow. The supersonic beam nozzle is an all-ceramic nozzle with either a $100-\mu \mathrm{m}$ pinhole in a watch jewel [19] or a 1-mm-long channel that has a diameter of $105 \mu \mathrm{m}$ drilled in alumina (Kulicke and Soffa-Israel). Both the gas mixing zone and the nozzle are separately heated. The carrier gas mixture and the sample molecules expand through the nozzle (5) (in the upper scheme), into the first vacuum chamber, which is pumped by a 4-in. diffusion pump [Varian VHS 4 (Varian Associates, Lexington, MA) backed by a $550-\mathrm{L} / \mathrm{min}$ rotary pump]. The emerging supersonic free jet is skimmed and differentially pumped $(63-\mathrm{mm}$ diffusion pump) and enters the electron impact ion source (6) in the high vacuum mass spectrometry chamber (63-mm diffusion pump). The nozzle can be aligned ( $X Y Z$ ) with respect to the skimmer from outside the vacuum chamber to maximize the beam intensity as measured by the increase of pressure in the mass spectrometry chamber.

The supersonic molecular beam is ionized by the fly-through Brink-type [20] EI ion source (6), which is open both at the entrance and exit of the SMB. The ionization cage is $25 \mathrm{~mm}$ long and $5 \mathrm{~mm}$ in diameter. The filament is made of a $0.2-\mathrm{mm}$-diameter thoriated tungsten wire purchased from Vacuumetrics. The ions are extracted by the ion optics attached to the EI ion source and guided by $90^{\circ}$ deflection (7) into a quadrupole mass analyzer (8) [VG-SXP 600 (VG Analytical Ltd., Manchester, UK)]. This quadrupole mass analyzer does not have a prefilter element and exhibits a significant reduction in ion transmission with mass. We measured an exponential transmission reduction factor of about 60 between masses 69 and 502 under conventional unit mass resolution conditions. The original commercial EI ion source was removed from the mass analyzer and its entrance contains an ion optics lens system.

Between the quadrupole mass analyzer and the channeltron ion detector there is a circular plate with a 3-mm hole denoted "exit lens". This plate is externally voltage biased to filter out ions produced from the thermal molecules of the vacuum background, and it transfers only ions produced in the hyperthermal SMB, which are distinguished by their higher kinetic energy $[2,3,16,17]$. As mentioned, in addition to eliminating the vacuum background, the background-free EI ion source facilitates ultrafast response time and exposes the mass spectrum of vibrationally cold molecules.

The SMB also can be square wave modulated by a beam chopper (9), which can be inserted into the beam trajectory or removed. This beam modulation allows the use of lock-in amplification for background subtraction when conventional EI is used.

The $90^{\circ}$ ion deflector can also be moved upward on an $X Y Z R$ vacuum manipulator and be replaced by a suitable surface for hyperthermal surface ionization (HSI) $[3,21,22]$ of the hyperthermal beam species.

Compatibility with standard GC was investigated by using a conventional gas chromatograph [Carlo Erba (Milan, Italy) Vega 6000] coupled to the fast gas chromatograph via a homemade, separately heated, transfer line. The GC column (15-m megabore J \& W DB-1; $1.5-\mu$ film thickness) ended inside the injector near the fast GC column, which served as a transfer line at that time by heating it to above the conventional GC column temperature. We used the Shrader System TM as the GC-MS software and for most of the data presentation.

Some additional important dimensions are the nozzle-skimmer distance, which is $5-8 \mathrm{~mm}$, the skimmer-surface (or ion deflector) distance of $\sim 60$ 
$\mathrm{mm}$, the beam diameter of $\sim 3.5 \mathrm{~mm}$ in the $\mathrm{EI}$ ion source, and the $90^{\circ}$ ion deflector of $15 \mathrm{~mm}$ from the quadrupole mass spectrometer entrance lens.

\section{Vibrational Cooling Effect on the Electron Impact-Mass Spectrometry of Octacosane}

Vibrational temperature effects on EI-induced ion fragmentation are well known [23-28] including in long chain alkanes [29]. In many books on mass spectrometry, at least one example of the pronounced effect of the ion source temperature on the degree of ion fragmentation appears [30,31]. The subject of low electron energy, low temperature EI-MS is reviewed by Maccoll and references therein [32].

Octacosane $\left[\mathrm{CH}_{3}\left(\mathrm{CH}_{2}\right)_{26} \mathrm{CH}_{3}\right]$ is a relatively long straight chain alkane molecule that has 86 atoms. It is a solid at room temperature and has a boiling point of $705 \mathrm{~K}$. Octacosane, like all the other long chain alkanes, is known to exhibit a mass spectrum that consists of low mass fragment ions without a molecular weight peak. The high temperature limit of the vibrational heat capacity of octacosane is $11.4 \mathrm{eV}$ at an ion source temperature of $250{ }^{\circ} \mathrm{C}$, whereas it is estimated, based on model calculations [33], that the actual heat capacity is about $3.5 \mathrm{eV}$. This intramolecular vibrational energy, together with the electron imparted vibrational energy, is sufficient to break the majority of all molecular ions. In a supersonic molecular beam, however, we anticipated that this thermal contribution would be practically eliminated. In Figure 2 the electron impact mass spectra of octacosane are shown. In trace a, the $18-\mathrm{eV}$ electron impact mass spectrum is plotted. In trace $b$ the 70-eV mass spectrum is shown. The lower trace (c) was taken from the NIST mass spectra library and it represents the standard 70-eV EI mass spectrum of thermal molecules at unspecified ion source temperature (probably in the range $150-250^{\circ} \mathrm{C}$ ). In contrast to trace $b$, trace $c$ does not show any molecular weight peak nor does it show any other high mass fragment peaks. In fact, the molecular weight peak does not exist in the library list of ions even at the level of $0.1 \%$. On the other hand, the 70-eV EI mass spectrum in a supersonic molecular beam is characterized by a dominant molecular weight peak that is the most prominent feature in the mass spectrum. The expanded mass scale near the molecular weight shows that all the molecular weight peaks exist, and their relative heights reflect ${ }^{13} \mathrm{C}$ natural abundance of $1.08 \pm 0.02 \%$, which is in good agreement with the literature value of $1.10 \pm$ $0.03 \%$. Thus, one can obtain the elemental information directly from the relative peak height ratios and determine the empirical formula. We note that the mass 394 $\mathrm{u}$ reflects the genuine molecular weight without any chemical ionization-related process of proton transfer.

We used a library search to identify the mass spectrum of trace $b$ by eliminating its molecular weight peaks and by limiting the search to molecules that had

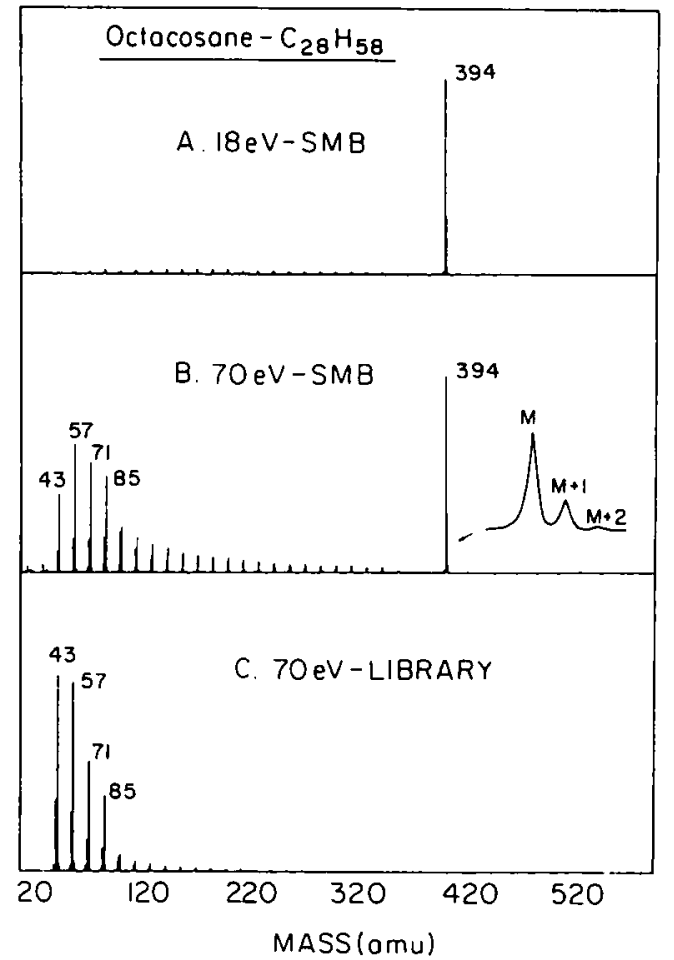

Figure 2. EI mass spectra in SMB of octacosane and a comparison with the NIST library thermal $70-\mathrm{eV}$ mass spectrum (trace $\mathrm{c}$ ). Trace a was obtained in SMB with 18-eV electron energy. Trace $b$ was obtained with $70-\mathrm{eV}$ electron energy.

a molecular weight of $394 \mathrm{u}$. Octacosane and two of its isomers appeared to be the best three candidates. On the other hand, we failed to locate octacosane through the mass spectrum obtained from an ion trap GC-MS [Finnigan ITD model 8100 (Finnigan-MAT, San Jose, CA)] with the standard library search algorithm. These search results demonstrate that when the molecular weight peak is a prominent mass spectrometry peak, the chances to obtain high confidence level library search results are increased. This observation emerges from the existence of all the usual fragments with only small changes in their relative peak heights. This result is rationalized by noting that the molecular weight ion can only dissociate, whereas all other peak heights can be increased by the dissociation of a higher mass peak and can be decreased by their own fragmentation. Therefore they are in a quasi-steady state. The molecular weight peak in octacosane is increased by more than 3 orders of magnitude compared with thermal 70-eV EI mass spectrum, whereas the peak heights from other fragments changed only slightly.

Because in SMB the thermal intramolecular vibrational energy is removed, the electron energy is the only parameter that governs the degree of fragmentation. The effect of the electron energy on the degree of fragmentation of the molecular weight ion is well known. However, the effect of reduced electron energy to achieve a noticeable increase in the relative molecular weight peak height also is associated with a consid- 
erable reduction in the EI ionization cross section. We have measured an EI ionization cross section reduction factor of 8 at $18 \mathrm{eV}$, in comparison with $70-\mathrm{eV}$ electron energy. In trace a we show that at $18 \mathrm{eV}$, the EI mass spectrum in SMB is dominated almost totally by the molecular weight peaks, whereas all other fragment peak heights are less than $5 \%$ of the normalized scale. More important is the observation that this considerable simplification of the mass spectrum was achieved where the absolute height of the molecular weight peak was reduced only by a factor of 1.5 . This observation results from the demonstrated degree by which the molecular ion dissociation is reduced, which compensates for the reduced ionization cross section.

We believe that the ability to achieve EI mass spectra that consist almost entirely of the molecular weight peak, without losing sensitivity, is of considerable importance. If any molecule in a complex mixture exhibits a single or very few mass spectral peaks, this could considerably extend the ability of GC-MS to handle its analysis in complex mixtures, because both the mass spectrometer and the gas chromatograph could serve as orthogonal separation tools with simplified deconvolution of overlapping GC peaks.

In addition, because the electron energy alone determines the degree of fragmentation of the various ions, this dependence can be explored and monitored. In Figure 3 we plot the normalized abundance of the indicated fragment and molecular weight ions versus the electron energy. Each ion peak height was normalized to its maximum value in the range of $10-80 \mathrm{eV}$. The electron energy was scanned by programming the electron energy power supply through a slow ramping

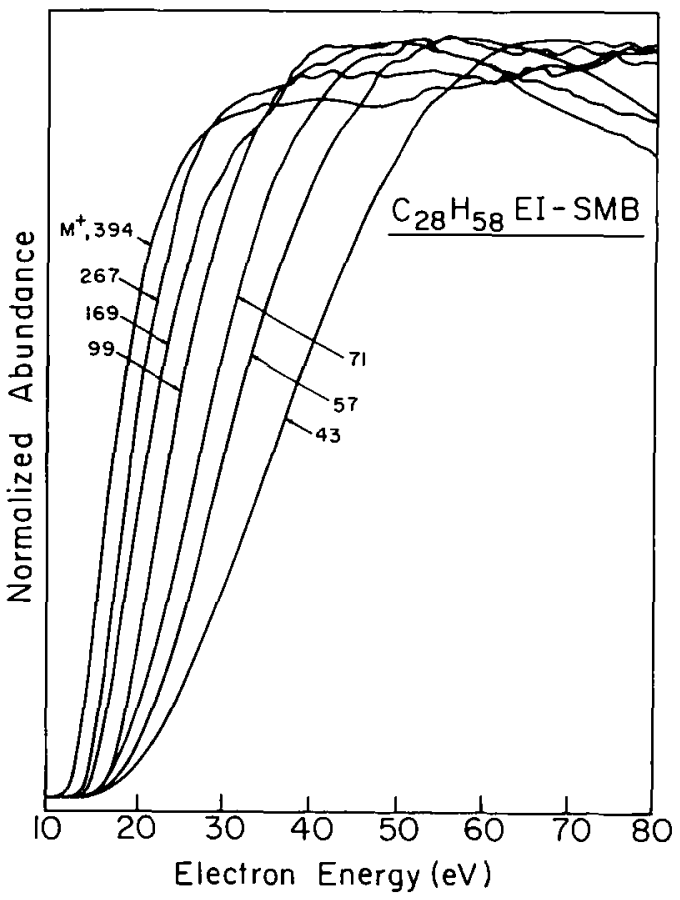

Figure 3. The dependence of the normalized octacosane fragment ion peak heights on the electron energy. oscillator. In Figure 3 it is observed clearly that each ion has a different normalized electron energy dependence. We also observe that the smaller the ion fragment is, the weaker is its dependence on the electron energy. This observation suggests that fragmentation occurs via a chain of fragmentation events, each to a close, but lower mass. It is believed that the measurements outlined in Figure 3 contain structural and energetic information, but this subject is beyond the scope of this article.

\section{Effects of the Length of the Alkane Chain on its Mass Spectrum in Supersonic Molecular Beams}

Because the vibrational heat capacity depends approximately linearly on the number of atoms in the molecule, one can expect an increase of the vibrational cooling effect with the length of the alkane chain.

We have studied the mass spectra of alkanes $\mathrm{C}_{8} \mathrm{H}_{18}$ through $\mathrm{C}_{40} \mathrm{H}_{82}$, and in Figure 4 the $70-\mathrm{eV}$ EI mass spectra in SMB of the indicated straight chain alkanes are portrayed. All these mass spectra are characterized

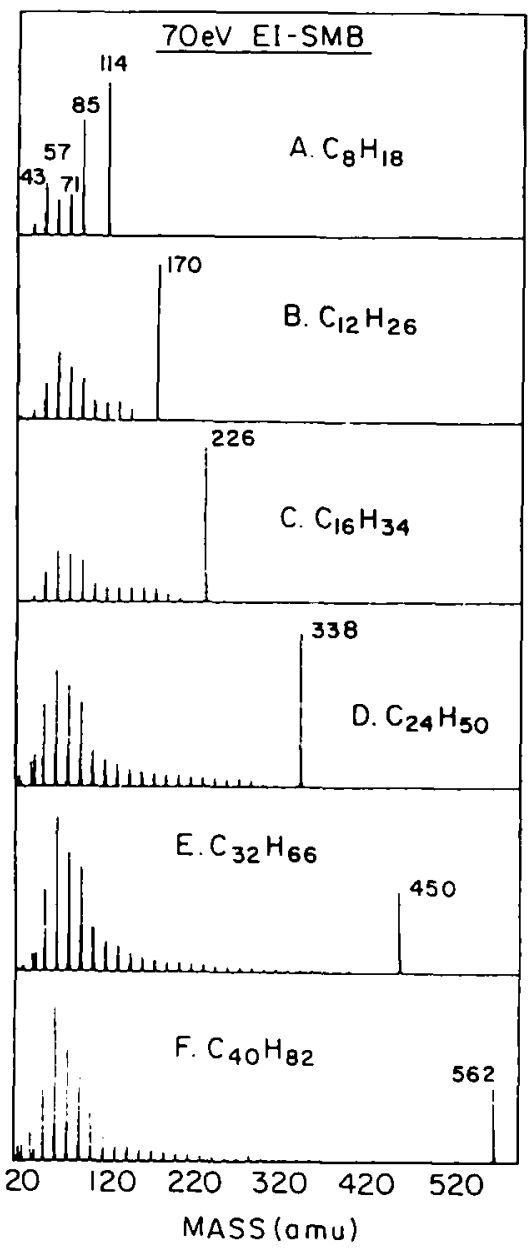

Figure 4. 70-eV electron impact ionization mass spectra in supersonic molecular beams of the indicated straight chain alkanes $\mathrm{C}_{8} \mathrm{H}_{18}-\mathrm{C}_{40} \mathrm{H}_{82}$. 
by a dominant or strong molecular weight peak. In fact, the molecular weight peak is always the strongest peak in the mass spectra. However, these mass spectra are distorted due to the substantial reduction in transmission at high masses of our quadrupole mass analyzer. In addition, a very long progression of peaks every $14 \mathrm{u}\left(\mathrm{CH}_{2}\right)$ is observed, which extends to fragments heavier than those in the library mass spectra of these compounds. Note that the library mass spectra also do not show a molecular weight peak for most of these alkanes.

It seems, therefore, that electron impact mass spectrometry in supersonic molecular beams exhibits the information content usually achieved by electron impact together with chemical ionization (CI) mass spectra in one scan. However, CI provides a degree of selectivity against alkanes and in favor of compounds that have high proton affinity. Moreover, the molecular weight peaks in EI provide elemental and isotopic information through the relative $\mathrm{M}^{+},[\mathrm{M}+1]^{+}$, and $[\mathrm{M}+2]^{+}$peak height ratios.

In Figure 5 we show the EI mass spectra in SMB of the same alkanes as in Figure 4, obtained at $18-\mathrm{eV}$ electron energy. It is clear that as the length of the

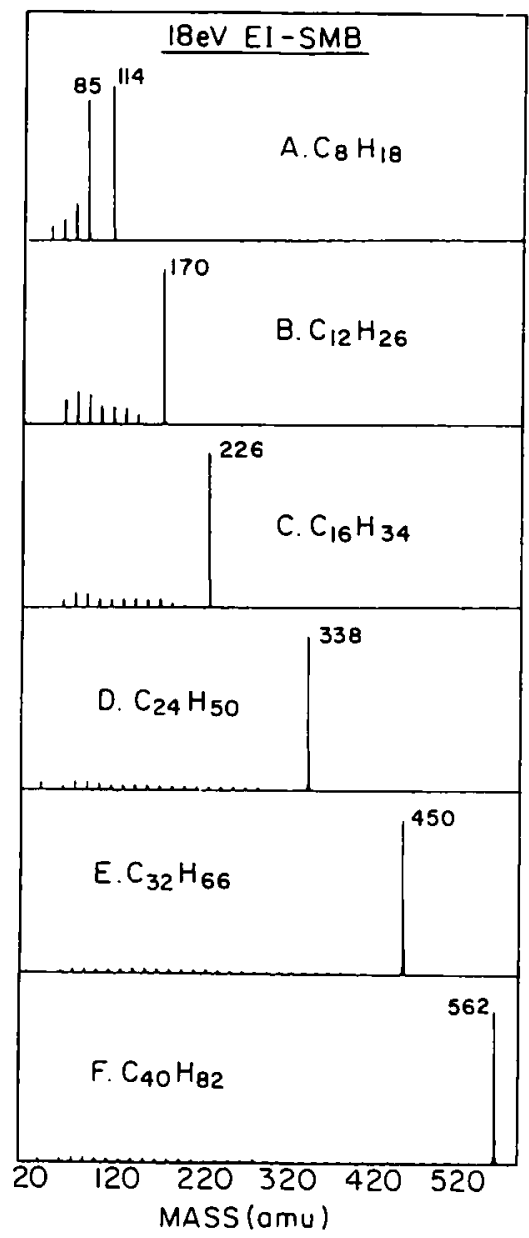

Figure 5. 18-eV electron impact ionization mass spectra in supersonic molecular beams of the indicated straight chain alkanes $\mathrm{C}_{8} \mathrm{H}_{18}-\mathrm{C}_{40} \mathrm{H}_{82}$. alkane chain increases, the 18-eV EI mass spectrum is dominated further by the parent undissociated molecular weight peak. For certain noncritical applications, one can say that above an alkane chain length of $\mathrm{C}_{16}$, the 18-eV EI mass spectra are essentially characterized by a single molecular weight peak and only small indication of other fragments. This claim is further supported by consideration of the strong transmission reduction of our quadrupole mass analyzer with mass.

As mentioned before, this simplification of the mass spectra at reduced electron energies also involves a reduction in the ionization cross section by a factor of 8 at $18 \mathrm{eV}$ in comparison with $70 \mathrm{eV}$ (these data were obtained by total ion current measurements normalized to the emission current in a DuPont magnetic sector mass spectrometer). However, this reduction in the ionization cross section is compensated by the substantially reduced degree of fragmentation encountered in EI mass spectra in SMB. In Figure 6, the reduction factor of the molecular weight signal at 18 $\mathrm{eV}$ in comparison with that of 70-eV EI mass spectra is plotted versus the chain length of the straight alkane. We note that the electron energy value is accurate to within $\pm 1.5 \mathrm{~V}$ due to a voltage gradient on the electron emission filament. From Figure 6 we observe that (1) the reduction of $\mathrm{M}^{+}$, and thus the reduction in detection sensitivity, is much smaller than the reduced ionization cross section by a factor of 8 ; (2) for alkanes with over 12 carbon atoms, this reduced sensitivity is less than a factor of 2 ; (3) the factor by which the sensitivity falls is reduced with the length of the alkane chain and approaches unity (no reduction) for long chain alkane molecules. We conclude therefore, that in SMB the simplification of the mass spectra that results from lowering the electron energy is achieved with a smaller penalty in sensitivity as compared with thermal electron impact mass spectrometry.

Comparison of Figures 4 and 5 indicates that although the effect of reduced electron energy on the mass spectra is relatively small in octane, it gradually increases with the length of the alkane chain. In Figure 7 , we plot the ratio of the molecular weight peak to the 57-u prominent fragment peak achieved at $18-\mathrm{eV}$ electron energy, divided by the same ratio achieved at an electron energy of $70 \mathrm{eV}$. This normalized ratio is a measure of the relative degree of control over the degree of fragmentation achieved by the electron energy. Note that this measure is normalized and unaffected by inconsistencies in the response of the quadrupole mass analyzer with the ion mass. Figure 7 demonstrates a purely exponential increase of the degree of control $(C)$ over the fragmentation achieved with increased chain length of the alkane. Although the mass spectrum of octane is hardly affected by the electron energy in the ranges $18-70 \mathrm{eV}$, where $C \approx 1.2$, the degree of control is increased to more than 50 for the $\mathrm{C}_{36}$ and $\mathrm{C}_{40}$ alkanes. The small deviation of $\mathrm{C}_{40} \mathrm{H}_{82}$ from the straight line is attributed to incomplete cooling in the SMB. 


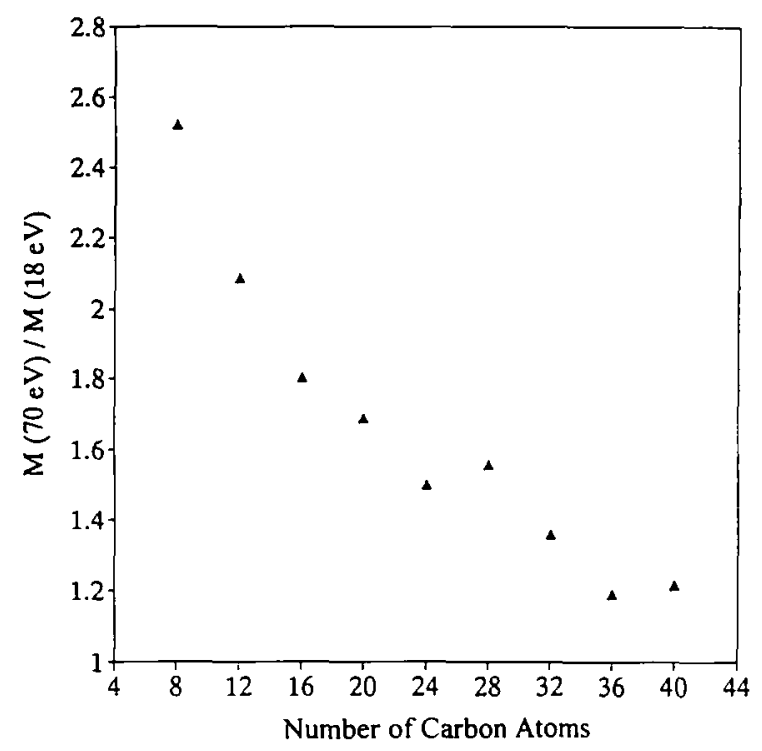

Figure 6. The ratio of the height of the molecular weight peak taken with 70- and 18-eV electron energy, plotted against the number of carbon atoms of straight chain alkanes $\mathrm{C}_{8} \mathrm{H}_{18}-\mathrm{C}_{40} \mathrm{H}_{82}$.

The observation shown in Figure 7 can be rationalized by using the following assumptions: (1) The vibrational energy given by the electron to the molecule is independent of the length of the alkane chain both at 18- and 70-eV electron energies (localized ionization); (2) the amount of intramolecular vibrational energy is negligible and can be ignored in comparison with the vibrational energy imparted by the electrons. By using these reasonable assumptions one finds that the amount of vibrational energy is molecular size independent,

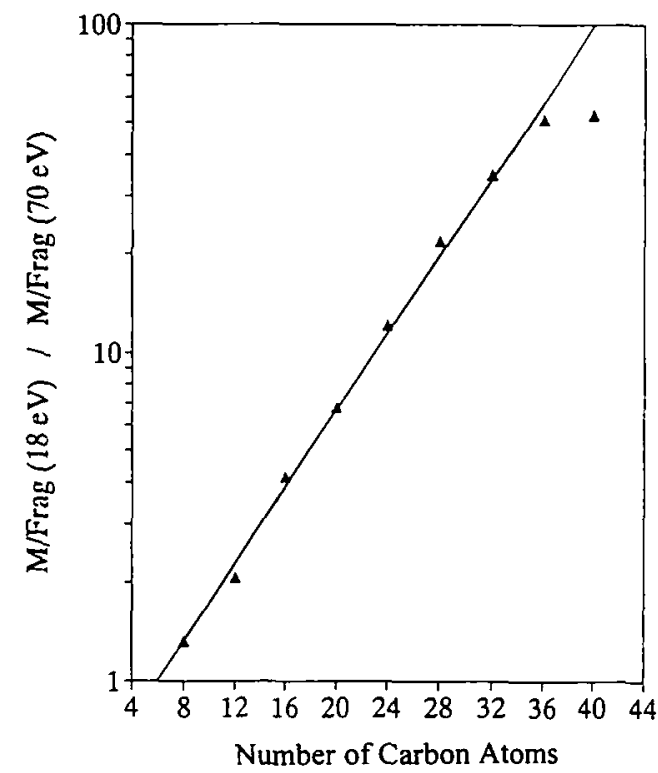

Figure 7. The ratio of the molecular weight peak divided by the $57-\mathrm{u}$ fragment peak height achieved at 18- and 70-eV electron energies, plotted against the number of carbon atoms of straight chain alkanes $\mathrm{C}_{8} \mathrm{H}_{18}-\mathrm{C}_{40} \mathrm{H}_{82}$. but the amount of vibrational energy per atom is reduced linearly with the number of atoms (chain length).

Accordingly, as the molecular size increases, its dissociation to small fragments such as the 57-u fragment is less likely, especially in view of the consecutive fragmentation processes implied from the results shown in Figure 3. If we further assume an exponential dependence of the rate of ion fragmentation on the excess vibrational energy and number of atoms, the degree of fragmentation will be reduced exponentially with reduced intramolecular vibrational energy content per atom at lower electron energy. In addition, the ratio of these reduction ratios also will behave in an exponential fashion as is demonstrated in Figure 7. Although the foregoing conjectured explanation may not be based on theoretically solid arguments, our experimental results clearly demonstrate increased control over the degree of molecular ion fragmentation with electron energy for larger molecules. We note that the extrapolation of our measurements to larger alkanes should result in the appearance of the molecular ion exclusively even at $70-\mathrm{eV}$ electron energy. In fact, if we correct our results for the reduced sensitivity of our quadrupole mass analyzer with increased mass, the molecular weight of $\mathrm{C}_{40} \mathrm{H}_{82}$ at $562 \mathrm{u}$ will be higher than all other fragments by a factor close to 20 in the $70-\mathrm{eV}$ electron energy mass spectrum.

Up to now we have addressed the question of the vibrational supercooling effect on the EI mass spectra of straight chain alkane molecules. In Figure 8 the 70-eV EI mass spectrum in SMB of the straight chain triacontane molecule $\left(\mathrm{C}_{30} \mathrm{H}_{62}\right)$ is compared with that of its branched isomer squalane. Pronounced isomer mass spectral differences are observed and new high mass major peaks appear through the enhanced fragmentation at the branching points that form secondary ions. A closer examination of the NIST library mass spectra of these molecules reveals that these isomer effects exist even in the thermal mass spectra, but they are considerably amplified in the EI-MS of the vibrationally cold molecules in the SMB. The mass spectrum of squalane contains relatively abundant ions at 182 , 238, 266, and $337 \mathrm{u}$ as well as neighbors to these masses. These ions are not straightforward cleavages of the carbon chain and are hard to explain. Again all these ions appear in the NIST library, whereas in SMB their relative abundance is largely amplified. In Figure 9 the 70-eV EI mass spectra in SMB of the indicated alkane derivatives are shown. Figure 9 demonstrates that the feature of a considerably enhanced molecular weight peak is universal to all molecules. In fact, the general trend we observed in these and many other molecules $[2,3,15,20]$ is that the smaller the molecular weight peak is in thermal 70-eV EI-MS, the larger is the enhancement achieved by vibrational cooling in SMB. Methylstearate exhibits a $21 \%$ molecular weight peak in the library (NIST) thermal 70-eV EI mass spectra. In the SMB its relative peak height is increased 
$70 \mathrm{eV} \mathrm{EI-SMB-MS} \mathrm{of} \mathrm{C}_{30} \mathrm{H}_{62}$

A. Triacontane
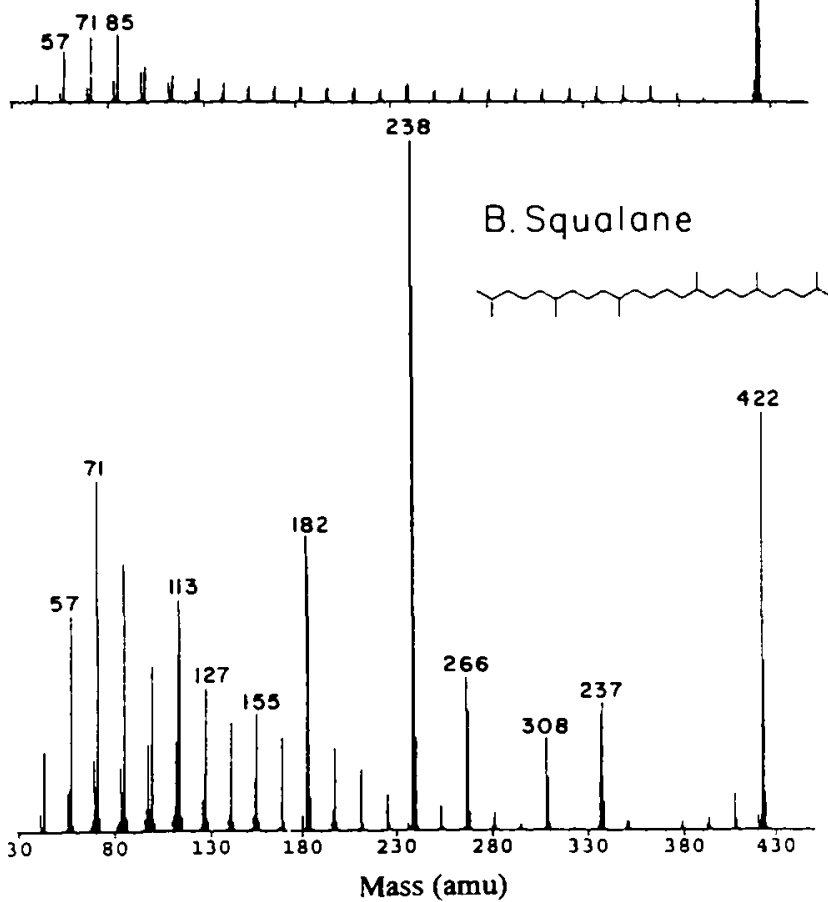

Figure 8. $70-\mathrm{eV}$ electron energy EI mass spectra of the straight chain triacontane (a) and its branched isomer squalane (b).

by about 1 order of magnitude. 1-Chlorohexadecane and 1-bromohexadecane do not show any molecular weight peaks in their thermal $70-\mathrm{eV}$ EI mass spectra and thus an enhancement of at least 3 orders of magnitude in peak height is observed. Dioctylphthalate in SMB exhibits only a $4 \%$ molecular weight peak, which is the lowest we have measured to date. We have studied the 70-eV EI mass spectrum of dioctylphthalate in a DuPont magnetic sector instrument and found that at an ion source temperature of $250^{\circ} \mathrm{C}$, the relative height of its $\mathrm{M}^{+}$peak is $\sim 2 \times 10^{-6}$ of the 149 -u peak, and that the $[M+1]$ peak is higher than expected, probably due to the ionization of dimers or due to residual chemical ionization. Thus, the small $\mathrm{M}^{+}$peak of dioctylphthalate in EI-SMB represents an increase of over 4 orders of magnitude in the relative height of the molecular weight peak.

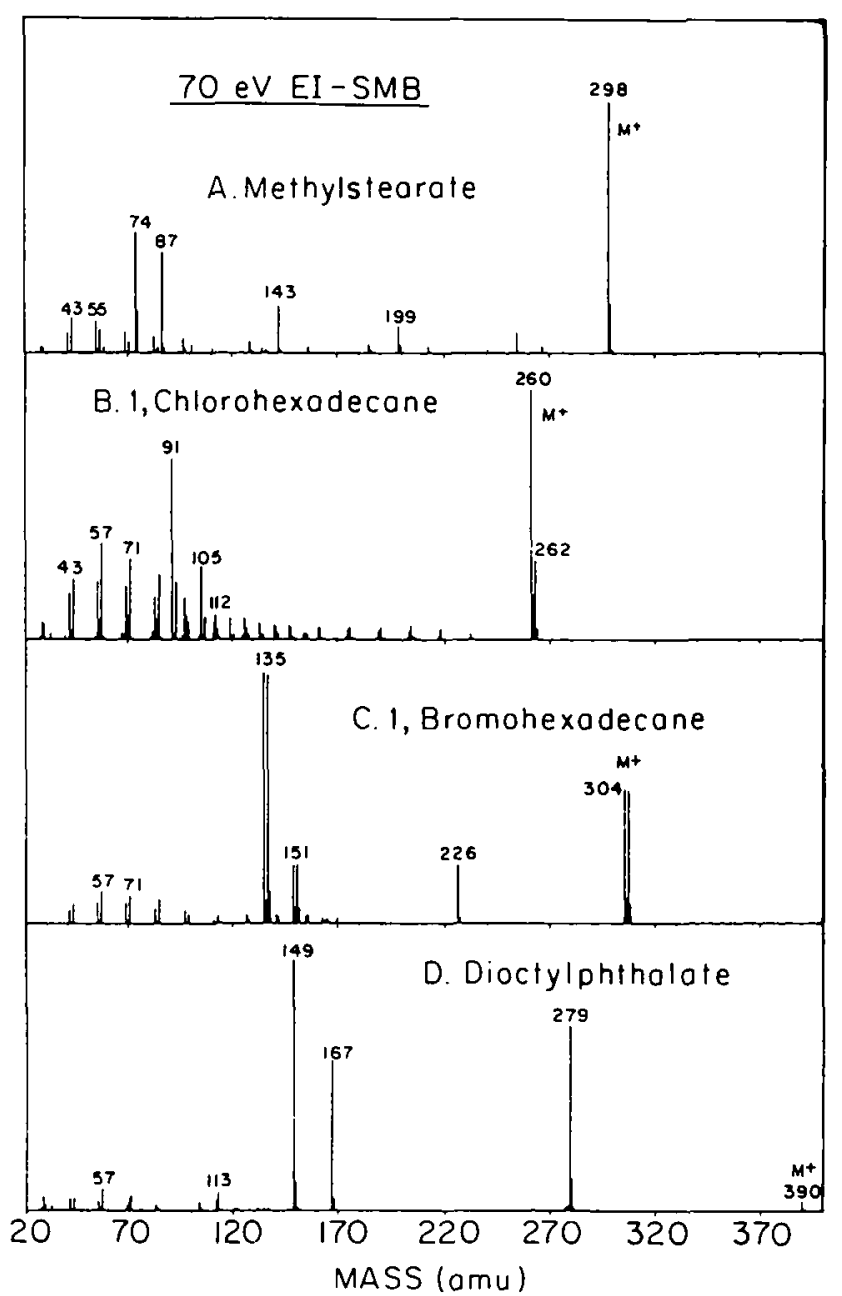

Figure 9. 70-eV electron energy EI mass spectra in SMB of the indicated alkane derivatives.

\section{Tradeoff of Response Time, Sensitivity, and Relative Molecular Weight Peak Height}

The effect of the vibrational temperature on the degree of molecular ion fragmentation is a very well known and established phenomenon [23-33]. Reduction of the ion source temperature indeed improved the molecular ion peak appearance, but involved a tradeoff of lowered ion source response time and reduced cleanliness. The relative height of the molecular weight peak also can be increased through the use of a better mass analyzer. A magnetic sector, in contrast to a quadrupole mass analyzer, does not exhibit a marked reduction in sensitivity with increased mass and enhances the apparent $\mathrm{M}^{+}$peak height. Another way to increase the relative height of the molecular weight peak is to reduce the electron energy with the tradeoff of detection sensitivity. In Figures 10 and 11 we demonstrate these three methods and discuss the tradeoffs involved. In Figure 10 mass spectra of octacosane $\left(\mathrm{C}_{28} \mathrm{H}_{58}\right)$ measured by a magnetic sector mass spectrometer are shown. They were taken at the indicated 


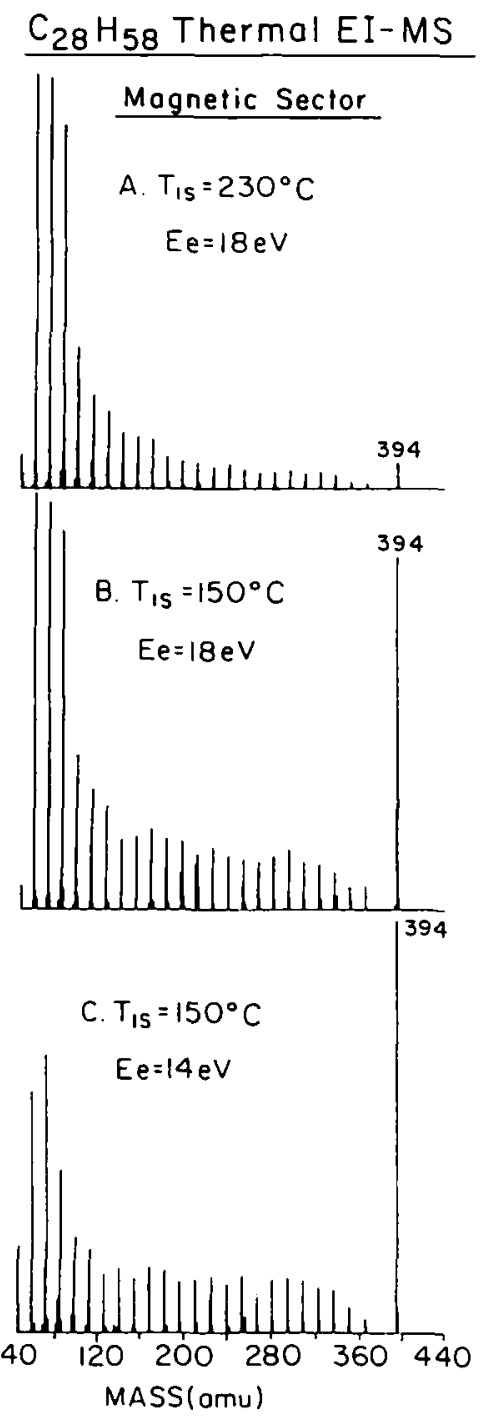

Figure 10. Electron impact ionization-magnetic sector mass spectrometry of octacosane achieved with a thermal ion source at the indicated ion source temperatures and electron energies.

electron energies and ion source temperatures. In contrast to the library 70-eV EI mass spectra, the magnetic sector instrument does provide a $6 \%$ normalized molecular weight peak at $18-\mathrm{eV}$ electron energy and an ion source temperature of $230^{\circ} \mathrm{C}$ as shown in trace a. This increased $\mathrm{M}^{+}$peak is the result of the flat mass response of the magnetic sector mass analyzer as well as the effect of reducing the electron energy to $18 \mathrm{eV}$. However, the tradeoffs are both in price and complexity through the use of a magnetic sector instrument instead of a quadrupole one and reduction of the ionization cross section (sensitivity) at the reduced electron energy by a factor of 8 . We note that reduced electron energy cannot be achieved with an ion trap mass spectrometer unless an external ion source is used. In trace $b$, the effect of the ion source temperature is demonstrated by its reduced temperature from 230 (trace a) to $150{ }^{\circ} \mathrm{C}$. The molecular weight peak is now strongly increased to about $90 \%$ of the normal-

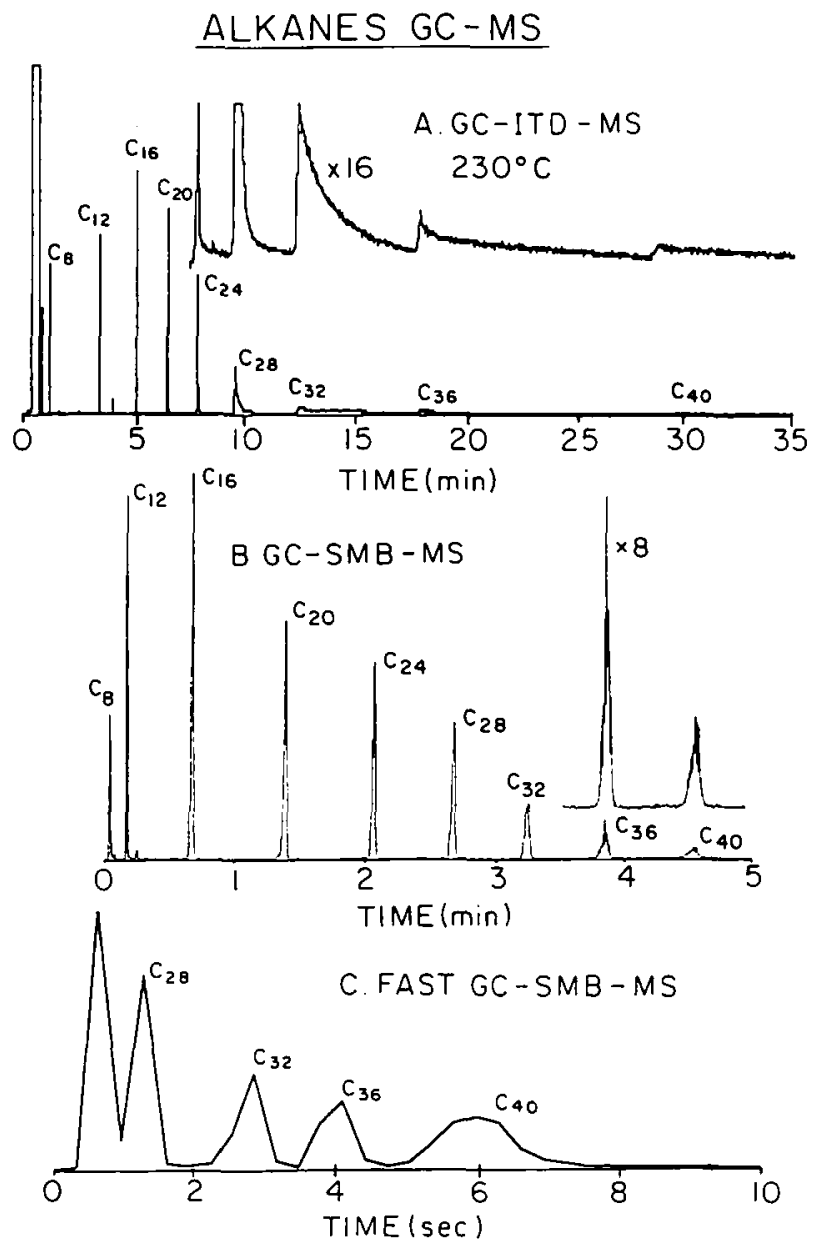

Figure 11. GC-MS and fast GC-MS of a mixture containing $\mathrm{C}_{8} \mathrm{H}_{18}-\mathrm{C}_{40} \mathrm{H}_{82}$ alkanes achieved using the following instruments: (a) Commercial Finnigan model 8100 ion trap gas chromatograph-mass spectrometer with a $30-\mathrm{m}$ narrow bore capillary column. (b) Supersonic molecular beam mass spectrometry combined with a gas chromatograph with a $4-\mathrm{m}$ narrow bore column (injection at $100^{\circ} \mathrm{C}$ and ramping at $49^{\circ} \mathrm{C} / \mathrm{min}$ to $300^{\circ} \mathrm{C}$ ). (c) Fast GC-MS in a supersonic molecular beam, achieved with the fast gas chromatography interface shown in Figure 1.

ized scale. This is achieved together with the additional penalty that at $150{ }^{\circ} \mathrm{C}$ octacosane cannot be quantified with GC-MS due to a severe loss of ion source response time and associated peak tailing. In trace $c$ the magnetic sector mass spectrum of octacosane was measured at an ion source temperature of $150{ }^{\circ} \mathrm{C}$ and 14-eV electron energy. At this electron energy the ionization cross section is lower than at 70 $\mathrm{eV}$ by a factor of 30 , but now the apparent mass spectrum seems similar to that of octacosane achieved with our quadrupole mass analyzer with 70-eV EI-MS in $\mathrm{SMB}$, as shown in Figure 2. Therefore, from Figure 10 it can be concluded that the use of supersonic molecular beams for $70-\mathrm{eV}$ EI-MS can provide mass spectra like those achieved by magnetic sector instruments at low electron energy and reduced ion source temperature, without the involved penalties of reduced sensitivity, much longer ion source response 
time, and increased complexity of the mass analyzer. Among these three features we believe that the ion source response time is the most significant because it concerns the mere ability to perform GC-MS experiments.

In Figure 11 we compare a total ion chromatogram obtained by GC-MS (Finnigan ITD 8100) with a conventional ion source and a chromatogram taken with supersonic molecular beam mass spectrometry. A mixture of $\mathrm{C}_{n} \mathrm{H}_{2 n+2}(n=8,12,16,20,24,28,32,36,40)$ was injected into the gas chromatograph. It is observed clearly that the ion trap response is characterized by extensive chromatographic peak tailing for the $\mathrm{C}_{24} \mathrm{H}_{50}$ and heavier alkanes. A closer examination revealed that even the eicosane $\left(\mathrm{C}_{20} \mathrm{H}_{42}\right)$ exhibited noticeable ion source-related peak tailing. As shown in the expanded scale $(\times 16)$, the extensive peak tailing renders GC-MS measurement of $\mathrm{C}_{32} \mathrm{H}_{66}, \mathrm{C}_{36} \mathrm{H}_{74}$, and $\mathrm{C}_{40} \mathrm{H}_{82}$ molecules impractical. In addition, the peak tailing found with $\mathrm{C}_{20} \mathrm{H}_{42}, \mathrm{C}_{24} \mathrm{H}_{50}$, and $\mathrm{C}_{28} \mathrm{H}_{48}$ results in a reduction of detection sensitivity and hampers quantitative measurement by considerably poorer reproducibility, as the exact amount of peak tailing may change due to long term thermal instabilities of the ion trap. Trace $b$ shows the EI-SMB GC-MS of the same alkane mixture by using a Carlo Erba Vega 6000 gas chromatograph with a 4-m narrow bore column $(250-\mu$ i.d. J \& W DB1; 0.25- $\mu$ film thickness). No peak tailing is observed although the chromatographic peak heights above hexadecane are reduced due to chromatographic peak broadening and due to the reduced response of the quadrupole mass spectrometer at higher masses. In Figure 11c we also demonstrate the ultrafast GC-MS [18] of a similar mixture of $\mathrm{C}_{24}-\mathrm{C}_{40}$ alkanes. In this case, with the help of flow programming after the elution of octacosane, the GC-MS trace ended after $8 \mathrm{~s}$ and even the $\mathrm{C}_{40} \mathrm{H}_{82}$ alkane molecule was characterized by a 1-s tail-free peak width. Thus, we conclude that, as expected from the use of background ion filtration, no tailing exists and the full temperature and volatility limit of the gas chromatograph can be exploited without mass spectrometric-related limitations. In addition, we note that we have examined the expanded mass spectra of hexadecane and eicosane obtained with the ion trap gas chromatograph-mass spectrometer and failed to find even a single ion at their molecular weight masses at the conventional ion source temperature used $\left(230^{\circ} \mathrm{C}\right)$.

\section{Detection Sensitivity with Electron Impact Mass Spectrometry in Supersonic Molecular Beams}

One of the most important parameters of any mass spectrometry ionization method is its associated detection limits. However, detection limits are usually quoted for a given molecule and experimental conditions that do not reveal the other interrelated parame- ters. Electron impact in supersonic molecular beams is different from conventional EI in several important aspects that affect the detection limits:

1. The supersonic molecular beam is inherently associated with jet separation, which reduces the molecular flux by a factor in the range of $2-20$. The heavier the molecule is, the smaller is the reduction factor. On the other hand, the very high flow $(240 \mathrm{~mL} / \mathrm{min})$ jet separator allows fast sampling of very large splitless injections and thus actually improves the achievable minimum detected concentration limit.

2. The molecules pass the ion source in fast motion only once instead of an estimated number of 10-20 cycles of intraion source adsorption-desorption rounds of thermal molecules which are 10 times slower. This means that the molecular ionization probability is smaller in the SMB by a factor of 100-200 times.

3. Because pyrolysis on the electron emitter filament and temperature gradients inside the ion source are not a problem, an open Brink-type ion source [20] is used with a filament close to the ion cage and with an electron emission current of up to $20 \mathrm{~mA}$. The considerably increased electron emission current and the high efficiency of the open Brink-type ion source, which allows several electron trajectories through it, compensate for most of the sensitivity losses due to the single fast molecular flight through the ion source.

4. The ion source ion optics and quadrupole mass analyzer transmission obviously affect the detection sensitivity. Typically, we detect less than $2 \%$ of the ions that exit the ion source at the channeltron ion detector (mostly quadrupole losses). The quadrupole transmission is further reduced exponentially at higher masses.

5. The vacuum background ion filtration process reduces the noise. This feature does not provide better detection limits, but rather allows peak performance near the detection limits to be achieved everyday regardless of the "history" of the apparatus. No vacuum system bake-out is needed to approach the minimum detection limit.

6. The supersonic molecular beam allows coupling of a fast gas chromatograph with narrower peak widths and thus higher peak flux, which contributes to improved detection limits.

7. The considerably enhanced molecular weight peak improves the single ion monitoring of chosen molecules through their molecular weight peak by a factor that can be over $10^{3}$.

Figure 12 demonstrates the detection sensitivity performance of our apparatus through the fast GC-MS of eicosane $\left(\mathrm{C}_{20} \mathrm{H}_{42}\right)$. The upper trace (a) demonstrates the single ion monitoring of eicosane through its molecular weight peak at $282 \mathrm{u}$. A solution of 100-ppb 

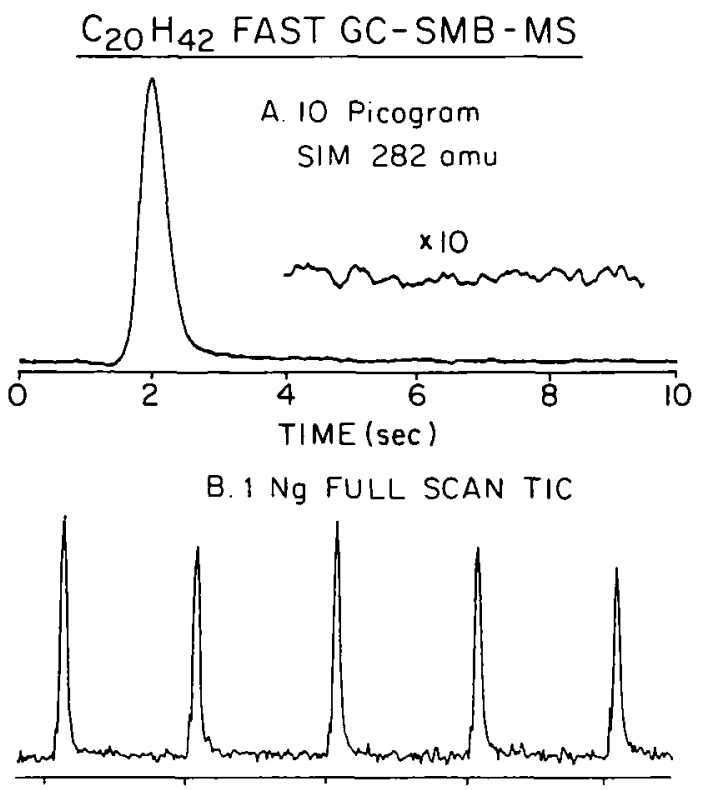

C. $1 \mathrm{Ng}$ FULL SCAN RSIM $282 \mathrm{omu}$

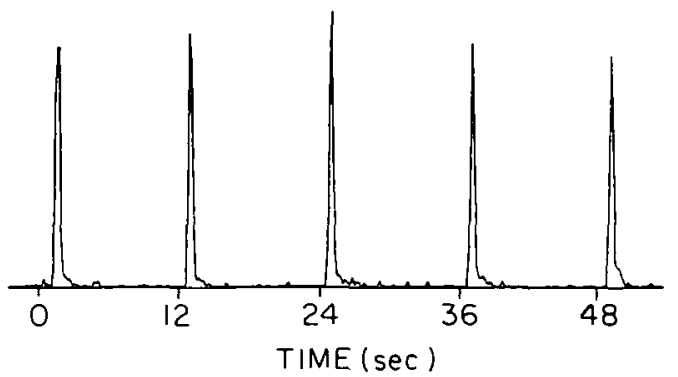

Figure 12. Sensitivity evaluation of EI-MS in SMB. (a) $0.1-\mu \mathrm{L}$ solution of 100-ppb eicosane (10 pg) was injected into the fast gas chromograph with the mass spectrometer tuned to the molecular weight mass of $282 u$. (b) Five repetitive $0.1-\mu \mathrm{L}$ injections, each after $12 \mathrm{~s}$, of a 10-ppm eicosane solution into the fast gas chromatograph-mass spectrometer. A full scan GC mass spectrum is shown with the mass spectrometer scanning. (c) As in b, but computer reconstructed single ion monitoring is shown at the molecular weight of $282 \mathrm{u}$.

eicosane in cyclohexane $(0.1 \mu \mathrm{L})$ was injected (splitless) into the $0.5-\mathrm{m}$ megabore column of the fast gas chromatograph inlet [18]. The GC-MS measurement was completed in $3 \mathrm{~s}$ and the observed chromatogram of $10 \mathrm{pg}$ of eicosane was practically noise-free. From the expanded $(\times 10)$ noise trace we estimate the signalto-two rms noise ratio to be 180 , and the linearly extrapolated minimum detected amount (MDA) to be $60 \mathrm{fg}$ (this value is $250 \mathrm{fg}$ if a three peak-to-peak noise value is considered). We believe this result is better than that which can be achieved with any other mass spectrometer for heavy alkanes by single ion monitoring at their molecular weight peak. We also have measured the signal by ion counting and found the overall signal to be about one molecular weight ion at the channeltron ion detector per femtogram of eicosane. The background noise count rate was 2000 ions per second and the rms noise fluctuations were about 25 ions per time constant of $0.3 \mathrm{~s}$. The noise originates predominantly from a mass-independent background ion signal, which depends on the total pressure. We believe this background signal originates from the insufficient ion filtration dynamic range of our quadrupole mass analyzer.

The lower trace (b) shows the full scan total ion chromatogram obtained by five consecutive injections of $1 \mathrm{ng}$ of eicosane $(0.1 \mu \mathrm{L}$, splitless, $10 \mathrm{ppm})$, each every $12 \mathrm{~s}$. The extrapolated MDA in this full scan mode is $30-40 \mathrm{pg}$. The lower trace (c) represents the same chromatogram as in trace $b$, but with a computer reconstructed single ion chromatogram at the molecular weight peak. In this case the detection limit is improved to below $10 \mathrm{pg}$. We note again that even with $100 \mathrm{ng}$ of eicosane we could not find even a single ion at the eicosane molecular weight in the conventional GC-MS shown in Figure 11. Thus, in this mode the SMB-MS is much more sensitive than an ion trap gas chromatograph-mass spectrometer. It should be mentioned also that when one searches for low detection limits, mass spectrometry in supersonic molecular beams also facilitates the addition of hyperthermal surface ionization $[3,21,22]$ which is a very sensitive ionization method with a demonstrated MDA of below $1 \mathrm{fg}$ [18]. However, hyperthermal surface ionization is relatively inefficient with alkanes and results in extensive molecular ion fragmentation. It is, however, an optimal ion source for the detection of aromatic molecules and amines that supplement the capability of the EI ion source and that is present in the same apparatus.

\section{Discussion and Overview}

We have shown that the EI-MS of alkanes in supersonic molecular beams is characterized by a substantial increase in the relative height of the molecular weight peak. The electron energy provides good control over the degree of molecular ion fragmentation with only a small sacrifice in the absolute signal of the molecular weight ion. In addition, the EI-MS in SMB provides tail-free GC-MS chromatograms without mass spectrometry ion source related restrictions on the molecular volatility.

As is well known and established, the enhanced molecular weight peak is of considerable importance for the following reasons:

1. This is a very important piece of information for detecting unknown molecules.

2. It helps to improve library searches.

3. This is the best mass spectrum peak to resolve overlapping gas chromatography peaks.

4. $\mathrm{M}^{+}$is the best peak for the single ion monitoring of target compounds.

5. The $\mathrm{M}^{+},[\mathrm{M}+1]^{+}$, and $[\mathrm{M}+2]^{+}$peak ratios provide elemental information. 
6. The existence of a noticeable molecular weight peak can provide isotope abundance geochemical information as well as enable deuterium exchange and isotope dilution analysis.

Obviously, all these aspects have long been attributed to EI-MS for those molecules that exhibit a pronounced molecular weight peak. The contribution of supersonic molecular beam sampling is merely in the extension of the range of molecules that exhibit molecular weight peaks. In this article we have demonstrated this for alkanes.

This work on EI-MS of alkanes in SMB is part of our continuous research on mass spectrometry in supersonic molecular beams. Our goal is to create a new mass spectrometric approach that will combine fast GC-MS, extended temperature limits, increased capability for handling thermolabile molecules, enhanced molecular weight peak height with the abovementioned features, possible selective ionization and increased sensitivity with hyperthermal surface ionization, possible coupling of gas chromatographs with any column and gas load, and the ability to use very large amounts of splitless injections.

\section{References}

1. Amirav, A.; Danon, A. U.S. Patent 5055677, 1991; Israel Patent 90970, 1989.

2. Amirav, A.; Danon, A. Int. J. Mass Spectrom. Ion Processes 1990, 79, 107.

3. Amirav, A. Org. Mass Spectrom. 1991, 26, 1.

4. Milne, T. A.; Greene, F. T. Adv. High Temp. Chem. 1969, 2, 107.

5. Pauly, H.; Toennies, J. P. Methods of Experimental Physics; Bederson, B.; Fite, W., Eds.; Academic: New York, 1968; Vol. 7A, p 227

6. Anderson, J. B.; Andres, R. P.; Fenn, J. B. Adv. Chem. Phys. 1965, 10, 275.

7. Kolodney, E.; Amirav, A. Chem. Phys. 1983, 82, 269; Danon, A.; Amirav, A. Rev. Sci. Instrum. 1987, 58, 1724.

8. Scoles, G., Ed. Atomic and Molecular Beam Methods; Oxford University Press: New York, 1988; Vol. 1.
9. Boesl, V.; Walter, K.; Schlag, E. W. Int. J. Mass Spectrom. Ion Processes 1986, 71, 309; Grotemeyer, J.; Boesl, V.; Walter, K.; Schlag, W. I. Am. Chem. Soc. 1986, 108, 4233; Org. Mass Spectrom. 1986, 21, 596.

10. Hager, J. W.; Wallace, S. C. Anal. Chem. 1988, 60, 5.

11. Lubman, D. M.; Jordan, R. M. Rev. Sci. Instrum. 1986, 56, 1299; Tembreull, R.; Lubman, D. M. Anal. Chem. 1986, 58, 1299; Tembreull, R.; Sin, C. H.; Pang, H. M.; Lubman, D. M. Optics News 1986, 12, 16

12. Leutuyler, S.; Even, U. Chem. Phys. Lett. 1981, 81, 578.

13. Meijer, G.; de Vries, M. S.; Hunziker, H. E.; Wendt, H. R. Appl. Phys. 1990, B51, 395; I. Chem. Phlys. 1990, 92, 7625.

14. Lubman, D. M., Ed. Laser and Mass Spectrometry; Oxford University Press: New York, 1990; Lubman, D. M. Mass Spectrom. Rev. 1988, 7, 535.

15. Amirav, A. J. Phys. Chem. 1990, 94, 5200.

16. Ballenthin, J. O.; Nier, A. O. Rev. Sci. Instrum. 1981, 52, 1016.

17. Nier, A. O.; Potter, W. E.; Kayser, D. C.; Fenslak, R. G. Geophys. Res. Lett. 1974, 1, 197.

18. Dagan, S.; Amirav, A. Int. J. Mass Spectrom. Ion Processes, 1994, 133, 187.

19. Danon, A.; Amirav, A. Rev. Sci. Instrum. 1987, 58, 1724.

20. Brink, G. O. Rev. Sci. Instrum. 1966, 37, 857 and 1626.

21. Danon, A.; Amirav, A. J. Phys. Chem. 1989, 93, 5549.

22. Danon, A.; Amirav, A. Int. J. Mass Spectrom. Ion Processes $1990,96,1390$

23. Danon, A.; Amirav, A.; Silberstein, J.; Salman, Y.; Levine, R. D. I. Phys. Chem. 1989, 93, 49.

24. Lifshitz, C.; Tiernan, T. O. J. Chem. Phys. 1973, 59, 6143; Lifshitz, C.; Weiss, M. Chem. Phys. Lett. 1972, 15, 266.

25. Chupka, W. A. I. Chem. Phys. 1971, 54, 1936.

26. Genuit, W.; Nibbering, N. M. M. Int. I. Mass Spectrom. Ion Processes 1986, 73, 61

27. Amorebieta, V. T.; Colussi, A. J. I. Phys. Chem. 1982, 86, 3056; 1986, 90, 1225; Chem. Phys. Lett. 1982, 89, 193.

28. Milne, T. A.; Beachey, J. E.; Greene, F. T. I. Chem. Phys. 1972, $56,3007$.

29. Remberg, G.; Remberg, E.; Spiteller, M.; Spiteller, G. Org. Mass Spectrom. 1968, 1,87.

30. Budzikiewics, H. In Biochemical Applications of Mass Spectromitry; Waller, G. R., Ed.; Wiley-Interscience: New York, 1972; p 251.

31. Davis, R.; Frearson, M. Mass Spectrometry; Wiley: New York, 1987.

32. Maccoll, A. Org. Mass Spectrom. 1986, 21, 601.

33. Turecek, F. Org. Mass Spectrom. 1991, 26, 1074. 